\title{
A Method of Recommendation of Points of Interest Based on Location Classification and User Similarity
}

\author{
Jennefer Mononteliza1)
}

\begin{abstract}
With the popularization of social network and global positioning technology, location-based social network applications have developed rapidly. Location-based social network data contains a lot of interest information. In order to solve the problem of poor recommendation result caused by the strong sparsity of matrix in traditional recommendation algorithm. This paper proposes a method of interest point recommendation based on location classification and user similarity. Social regularization is used to constrain the difference of location category preference in social relations, which solves the data sparsity problem of collaborative filtering. The proposed algorithm was tested using Foursquare public dataset and compared with the relevant algorithm. Experimental results show that the proposed algorithm has high precision and recall.
\end{abstract}

Keywords: Location-based Social Network, Social Network, Recommendation, Algorithm

\section{Introduction}

With the rapid development of mobile Internet and the continuous popularization of global positioning system, mobile terminal equipment has gradually become an indispensable electronic device in our daily life. This has greatly accelerated the rise of location-based social networks (LBSN), such as Twitter, Yelp, Foursquare, and so on. According to Foursquare's website, more than 50 million people use Foursquare each month. There are more than 12 billion location check-in every month. That's a staggering number, and at its peak it can generate more than 9 million check-in a day. This fully shows that location-based social networks have been widely promoted and used[1][2].

These location-based social networks encourage users to share their travel experiences and current location with friends in real time. These semantic locations are collectively referred to as POI (point-of-interest) (such as restaurants and cinemas). This mobile service, which allows users to share their location in real time, allows users' friends to fully participate in the

Received(December 14, 2019), Review Result(1st: January 15, 2020, 2nd: Marych 03, 2020), Accepted(April 25, 2020)

1) (Professor) University of Antique, Antique, Philippines

email: jenneferjmonteliza@gmail.com 
process, enhancing the friendship between friends and enriching the current social network atmosphere[3]. In general, a typical check-in record contains not only the text information posted by the user, but also the check-in time and location information such as latitude and longitude coordinates[4][5]. Users' personal preferences can be mined by collecting their check-in records on location-based social networks. To provide users with a series of location services that meet their preferences and needs, such as point of interest recommendations. At present, various social networking sites have generated a large amount of user check-in data, which is worth studying the user travel patterns included in them. These check-in data with semantics and coordinates can not only help users find interesting places in new areas, but also help third parties or merchants attract customers. Therefore, they are of high research value[4-7].

\section{Related Research}

\subsection{Recommendation of Points of Interest Based on Geographical Location}

The user interacts with the location through real-time check-in, and the distance between different check-in locations often reflects the user's behavior pattern in terms of geography. The distance between different locations can be characterized by power distribution and gaussian distribution. The paper [4] processed the public data sets of Foursquare and Whrrl, and found that the probability of users checking in at a certain place and the distance from that place to the current location presented a power-rate distribution. Paper [5] believes that users' check-in is usually around some regional centers, so a multi-center gaussian model is proposed. On the contrary, the paper [8] believes that different users have different behavior patterns for geographical factors, and it is not possible to use power ratio distribution or gaussian distribution to simulate them uniformly, while the kernel density model can realize user personalization.

\subsection{Recommendation of Points of Interest Based on Time Factor}

Users' check-in data usually show a certain time regularity, such as periodicity or continuity. In this paper [5], a day is divided into 24 hours, and the time-user-place $3 \mathrm{~d}$ matrix is constructed and the similarity between time is used to fill the $3 \mathrm{D}$ matrix, so as to improve the shortcomings existing in traditional collaborative filtering. Paper [9] also divided a day into 24 $h$, constructed user-place matrix for each time period, and then decomposed the matrix to 
obtain the preference under different time periods. The paper [6] believes that the check-in in a short period of time has a strong correlation, and the Markov chain can be used to simulate the sequence change to recommend the next location in the sequence.

\subsection{Recommendation of Points of Interest Based on Location Category}

Few studies consider the category of location, and categories can play an important role in some real-world situations. The paper [10] USES HITS algorithm to create "local experts" for each location category in different cities. When users visit a new city, they model user preferences and match corresponding "local experts" to find suitable recommended locations. After calculating the user's location preference, the paper [11] clustering users based on k-means algorithm, and then applying matrix decomposition technology to the user category matrix to obtain the next possible access category. At the same time, combined with the geographical model represented by power-rate distribution, the final recommendation results are generated. In the paper [12], users are divided into different groups and represented by location category check-in vector. Then, according to the geographical similarity between different groups, collaborative filtering method is adopted to complete the recommendation. The paper [13] believes that the user's rating of a site is irrelevant to the category of nearby sites, so the influence of nearby sites should be filtered out to complete the recommendation of target sites.

\subsection{Recommendation of Points of Interest Based on Social Networks}

With the rise of social networks in recent years, more and more research has focused on social relationships. Early on, the paper [14] proposed the use of social relations in collaborative filtering, because friends may go to the same places. The paper [15] proposed that friends would not check into the same place to a large extent, and the experiment also showed that the same place in the social relationship could not be effectively used to improve the accuracy of the recommendation result. Therefore, it is unreasonable to get location recommendation directly from friends. In the traditional recommendation system, most of the recommendation targets are single and simple online items such as movies and commodities, which do not have the physical property of real-time movement. 


\section{Recommendation of Points of Interest}

LBSN typically pre-defines the location category information, such as the location category in Foursquare, which is a three-tiered framework. The user category matrix is better than the user location matrix in sparsity, and the category can largely reflect the user's preference for a functional or semantic location. The accuracy of the recommendation can be improved by calculating the category preference difference based on the historical check-in data and then combining the category information to recommend users' interest points.

Compared with the traditional recommendation system, the algorithm proposed in this paper does not directly use the user location relationship to make recommendations, because this method will lead to poor recommendation results under the strong sparsity of the matrix. The algorithm in this paper firstly extracts the user category matrix $\mathrm{R}$ from the original data set and marks it with 0 and 1 . If user $i$ has visited a certain category $j$, the value of is 1 , and if not, it is 0 , and represent the total number of categories. Matrix decomposition is used and promoted by many recommendation system algorithms. We also use matrix decomposition to analyze the category matrix and obtain the user's feature matrix and the category's feature matrix. $f$ represents the feature dimension and can be selected according to experience. The loss in the decomposition process is

$$
\Gamma=\frac{1}{2} \sum_{|i=1|}^{|U|} \sum_{j=1 \mid}^{|C a|} I_{i j}\left(R_{i j}-P_{i}^{T} Q_{j}\right)^{2}+\frac{\lambda_{1}}{2}\left\|P_{i}\right\|_{F}^{2}+\frac{\lambda_{2}}{2}\left\|Q_{j}\right\|_{F}^{2}
$$

In the formula, I is the indicator function, whose function is to restrict the positions that have not been visited by the user, so that their weight is 0 , while the positions that have been actually visited by the user are 1 . and are the eigenvectors about user $I$ and category $j$ respectively. For matrix decomposition, regularization loss can effectively prevent overfitting, and is the weight of the regularization term, and represents the Frobenius norm. Finally, we continue to iterate and update the feature vectors of users and categories through the classical gradient descent algorithm. Usually, a location has more than one category, so it corresponds to the feature vectors of multiple categories. By integrating these category vectors, the feature vectors of the location can be obtained. First, mapping location and category is conducted to obtain the representation matrix of location and category. Similarly, the form of marking 0 and 1 is adopted. If a location belongs to a certain category, the corresponding element in the 
matrix is 1.0 is the place that doesn't matter in this category. Then, according to the site category matrix, the feature matrix $\mathrm{H}$ of the site can be obtained as

$$
H=Q M^{T}
$$

The score value of user $\mathrm{u}$ to unknown location 1 can be calculated by formula 3 . Where, $u$ $\mathrm{P}$ is the feature vector of user $\mathrm{u}$ updated according to formula (1), and $1 \mathrm{H}$ is the feature vector of location 1. Finally, all the scores are sorted to recommend Top-K points of interest to users.

$$
\text { Score }=P_{u}^{T} H_{L}
$$

Most current research on recommendation of points of interest considers friends' social relationships directly in terms of similarity. This article has shown that there are big differences in friends' preferences for the same places. Therefore, it is not appropriate for traditional recommendation systems to rely on the similarity between friends or to obtain the location directly from friends.

The site category is a refinement of the function and semantics of the site, which can reflect the user's personal preference. The Foursquare dataset shows that the proportion of co-check-in categories among users is much higher than that of co-check-in locations. Therefore, from this perspective, this paper proposes to use social regularization to model this kind of difference:

$$
\frac{\lambda_{3}}{2} \sum_{i=1_{o} \in F r e^{+}(i)}^{|U|} \sim(i, o)\left\|P_{i}-P_{o}\right\|_{F}^{2}
$$

In the formula $\lambda_{3}$ is the weight parameter of weighing the role of social regularization. The list of friends that user $\mathrm{i}$ actively follows in the social network; calculates the preferred similarity between user $\mathrm{i}$ and user $\mathrm{o}$ in the location category. There are many ways to calculate the similarity. The traditional method is cosine similarity. In this paper, the jackard coefficient is used.

$$
\operatorname{Sin}(i, o)=\frac{\left|C_{i} \cap C_{o}\right|}{\left|C_{i} \cup C_{o}\right|}
$$


Where, is all the categories that user $\mathrm{i}$ has visited. In the current research progress of recommendation system, social regularization has been recognized and used by many researchers. It alleviates the data sparsity problem of collaborative filtering and guarantees the quality of recommendation.

In this paper, we propose a point of interest recommendation algorithm that combines place categories with social networks. The overall framework is shown in [Fig. 1]. On the one hand, the user category matrix can alleviate the sparsity compared with the user location matrix. On the other hand, using social regularization to constrain the difference of category preference in social relations can make the algorithm more realistic. Formula 1 is the loss of updating parameters of gradient descent, and formula 4 is the loss of introducing social relations. In this paper, the total loss of the proposed algorithm is shown in formula 6, where all the parameters have been described in the previous section.

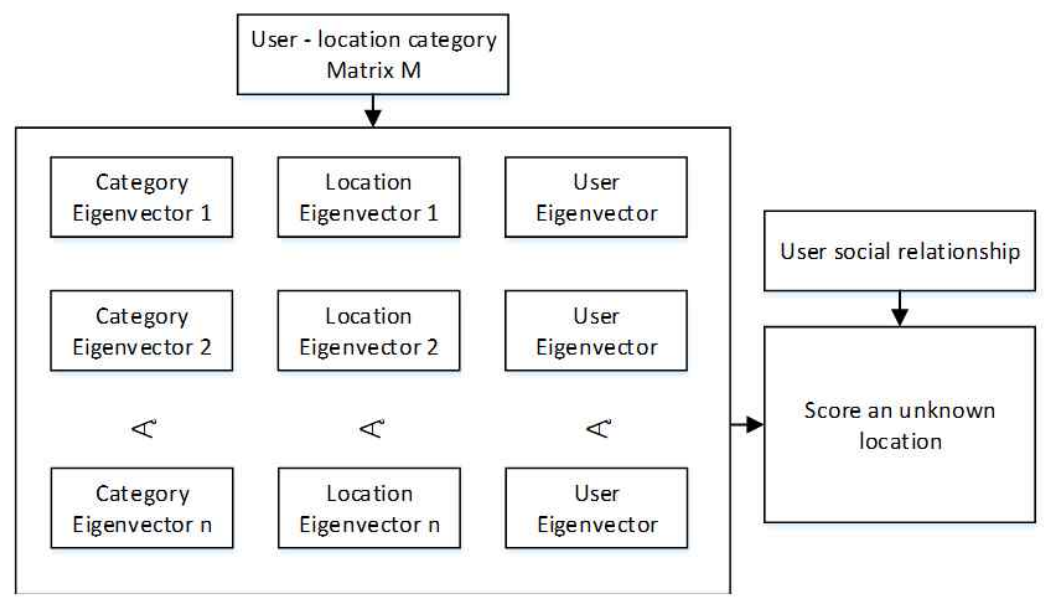

[Fig. 1] Overall Framework

Similarly, the stochastic gradient descent method is used to solve $\mathrm{P}$ and $\mathrm{Q}$ for the objective function, and formula 7 and formula 8 are the derivative formulas. Where, is a collection of friends who actively follow user I. After obtaining $\mathrm{P}$ and $\mathrm{Q}$, we can combine formula 2 and formula 3 to calculate the final score of user $u$ to unknown location 1. Finally, the scores of all places were sorted from high to low, and the Top-K points of interest were selected as the recommended results.

$$
\Gamma=\frac{1}{2} \sum_{|i=1| \mid}^{|U|} \sum_{j=1 \mid}^{|C a|} I_{i j}\left(R_{i j}-P_{i}^{T} Q_{j}\right)^{2}+\frac{\lambda_{1}}{2}\left\|P_{i}\right\|_{F}^{2}
$$




$$
\begin{gathered}
+\frac{\lambda_{2}}{2}\left\|Q_{j}\right\|_{F}^{2}+\frac{\lambda_{3}}{2} \sum_{i=1_{o} \in F_{r e} e^{+}(i)} \sim(i, o)\left\|P_{i}-P_{o}\right\|_{F}^{2} \\
\frac{\partial \Gamma}{\partial P_{i}}=\sum_{|j=1|}^{|C a|} I_{i j}\left(P_{i}^{T} Q_{j}-R_{i j}\right) Q_{j}+\lambda_{1} P_{i}+\lambda_{3} \sum_{o \in F r e^{+}(i)} \sim(i, o) \quad\left(P_{i}-P_{o}\right) \\
+\sum_{o \in F r e^{-}(i)} \sim(i, g)\left(P_{i}-P_{g}\right) \\
\frac{\partial \Gamma}{\partial Q_{i}}=\sum_{|i=1|}^{|U|} I_{i j}\left(P_{i}^{T} Q_{j}-R_{i j}\right) P_{j}+\lambda_{1} Q_{j}
\end{gathered}
$$

\section{Results}

\subsection{Dataset}

This paper uses the Foursquare dataset. Foursquare is a mobile and portable service that encourages users to share location information based on their location. This article selects data from September 2015 to September 2017, covering 162 cities, with a total of 33,44,759 check-in data. This paper selects a large number of check-in data from Stockholm. The data set was preprocessed to remove POI with less than 10 check-in Numbers and POI with less than 10 user check-in Numbers. The final data contains 205,358 check-in data.

\subsection{Evaluation Method}

In the recommendation system, the commonly used evaluation indicators are Precision (Ppre-@k) and recall (Rrec@k), which are also the most common indicators in the field of machine learning. Accuracy is the percentage of places that are correctly recommended in the list of recommendations generated for the user. The recall rate is the percentage of locations that are correctly recommended in the user's test set. The specific calculation formula of these two indexes is

$$
\begin{gathered}
P_{\text {Pre@K }}=\frac{1}{\left|U_{\text {test }}\right|} \sum_{u \in U_{\text {test }}} \frac{\left|L_{u}^{r e c} \cap L_{u}^{\text {test }}\right|}{K} \\
R_{\text {Rec@K }}=\frac{1}{\left|U_{\text {test }}\right|} \sum_{u \in U_{\text {test }}} \frac{\left|L_{u}^{r e c} \cap L_{u}^{\text {test }}\right|}{\left|L_{u}^{\text {test }}\right|}
\end{gathered}
$$


represents the test set user set. is the list of recommendations that our algorithm generates for users, while Lutest is the actual set of locations that user $u$ needs to predict in the test dataset. In the experiment, $\mathrm{K}$ values were 10, 20 and 50 according to most algorithms.

\subsection{Results}

The algorithm proposed in this paper fully combines the location category and social relations, aiming to improve the traditional classic recommendation model. Therefore, the comparison algorithm selected in this study includes the following:

\subsubsection{UserCF Algorithm}

The popular and classic user-based collaborative filtering algorithm calculates the similarity between users according to the user location matrix to complete the recommendation of target users.

\subsubsection{UC Algorithm}

It is a recommendation algorithm based on location category, without considering the social relationship, and its core formula can be obtained according to the solving process of location category score in this paper.

\subsubsection{Basic MF Algorithm}

recommendation algorithm based on matrix decomposition technology. the original user location matrix is decomposed to fill it to find potential unknown locations.

[Fig. 2] and [Fig. 3] show the experimental results of accuracy and recall rate. When the length of the recommendation list is 10, 20 and 50, respectively, the proposed algorithm is superior to other recommendation algorithms under the two evaluation indexes, which fully shows that taking place category and social relationship into consideration is conducive to improving the recommendation effect. It is worth mentioning that UC algorithm represents the intermediate process of proposed algorithm, that is, the recommended results under the influence of location category. However, compared with Basic MF algorithm and UserCF algorithm, its accuracy and recall rate still have obvious advantages, which also reflects the importance of location category. However, UC algorithm does not combine social relations. When the recommended number is 10, the proposed algorithm in this paper combining category and social relations is far more than UC algorithm. But the gap narrowed as the number of recommendations increased, suggesting that social connections had a limited effect. 
No matter in terms of accuracy or recall rate, the effect of UserCF is the worst. We analyzed that this may be caused by data sparsity. Building user location matrix directly will make the matrix rather sparse, so there are obvious defects in the calculation of user similarity, resulting in poor final recommendation effect.

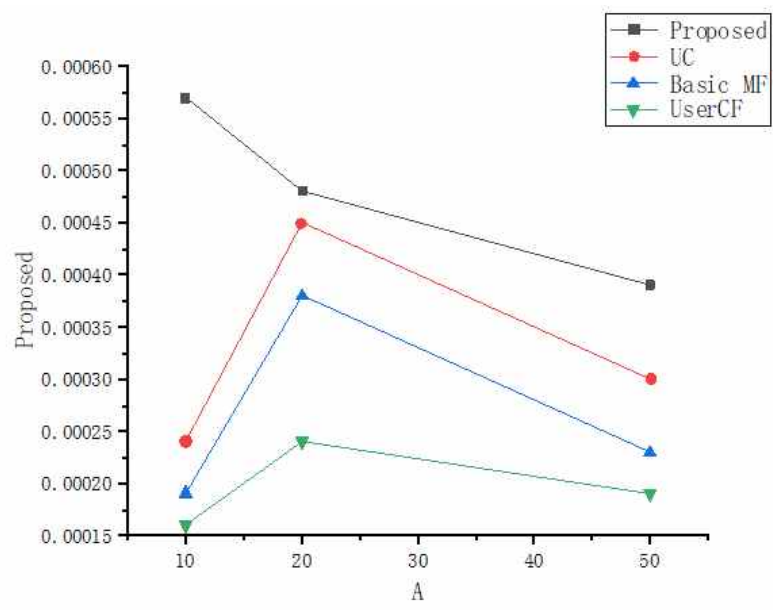

[Fig. 2] Precision

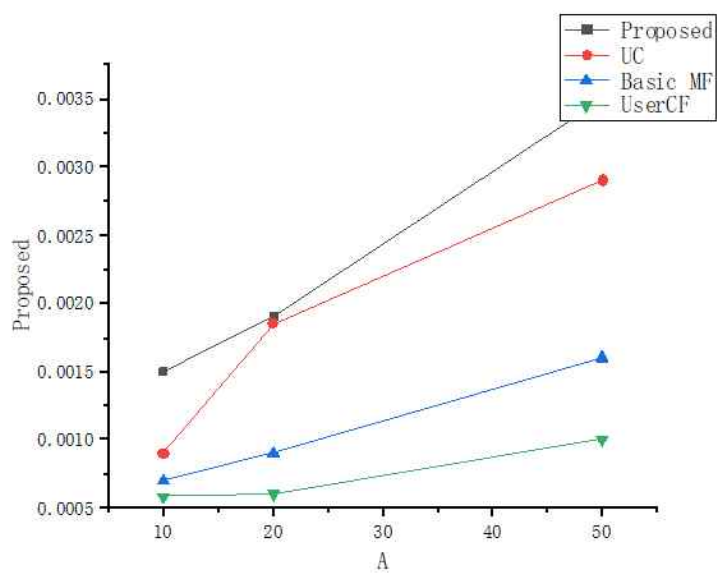

[Fig. 3] Recall

\section{Conclusion}

Point of interest recommendation is an important subfield in the recommendation system. This paper first summarizes the existing research work on recommendation of points of interest, and then proposes a recommendation algorithm based on place category and social relationship. On 
the one hand, the location category can better reflect the user's preference than the location itself, on the other hand, it can effectively alleviate the sparsity when building the matrix. The Foursquare dataset shows that far more friends visit the same categories than the same places. Therefore, the proposed algorithm is suitable for the research of point of interest recommendation. Finally, this paper compares with the current mainstream classical recommendation algorithm, and the experimental results prove the advantages and effectiveness of the proposed algorithm.

\section{References}

[1] Jinying Jia, Fengli Zhang, Non-deterministic k-anonymity algorithm based untrusted third party for location privacy protection in LBS, International Journal of Security and Its Applications, (2015), Vol.9, No.9, pp.387-400, DOI: http://dx.doi.org/10.14257/ijsia.2015.9.9.33

[2] Minho Cho, Response analysis model of social networks using fuzzy sets and system dynamics, International Journal of Grid and Distributed Computing, (2018), Vol.11, No.4, pp.1-12, DOI: http://dx.doi.org/10.14257/ijgdc.2018.11.4.01

[3] Zhao Z, Xu W, Xue C, Wang L, Mutual graph embedding in lbsn: capturing multi-factor influence for point-of-interest prediction, 2019 IEEE SmartWorld, Ubiquitous Intelligence \& Computing, Advanced \& Trusted Computing, Scalable Computing \& Communications, Cloud \& Big Data Computing, Internet of People and Smart City Innovation, (2019), August 19-23; Leicester, United Kingdom, DOI: 10.1109/SmartWorld-UIC-ATC-SCALCOM-IOP-SCI.2019.00211

[4] Ye M., Yin P. F., Lee W. C., Lee D. L., Exploiting geographical influence for collaborative point-of-interest recommendation, Proceedings of the 34th international ACM SIGIR conference on Research and development in Information Retrieval, (2011), July 24-28; Beijing, China, pp.325-334, DOI: https://doi.org/10.1145/2009916.2009962

[5] Yuan Q., Cong G., Ma Z., Sun A., Thalmann N., Time-aware point-of-interest recommendation, Proceedings of the 36th international ACMSIGIR conference on Research and development in information retrieval, (2013), July 28-August 1; Dublin, Ireland, pp.363-372.

[6] Feng S. S., Li X. T., Zeng Y. F., Cong G., Personalized ranking metric embedding for next new POI recommendation, Proceedings of the 24th International Conference on Artificial Intelligence, (2015), July 25-31; Buenos Aires, Argentina, pp.2069-2075.

[7] Griesner J. B., Abdessalem T., and Naacke T., POI recommendation: towards fused matrix factorization with geographical andtemporal influences, Proceedings of the 9th ACM Conference on Recommender Systems, (2015), September 16-20; Vienna, Austria, pp.301-304, DOI: https://doi.org/10.1145/2792838.2799679

[8] Zhang J. D., Chow C. Y., GeoSoCa: Exploiting geographical, social and categorical correlations for point-of-interest recommendations, Proceedings of the 38th International ACM SIGIR Conference on Research and Development in Information Retrieval, (2015), August 9-13; Santiago, Chile, pp.443-452, DOI: https://doi.org/10.1145/2766462.2767711 
[9] Gao H. J., Tang J. L., and Hu X., Liu H., Exploring temporal effects for location recommendation on location-based social networks, Proceedings of the 7th ACM conference on Recommender systems, (2013), October 12-16; Hong Kong, China, pp.93-100, DOI: https://doi.org/10.1145/2507157.2507182

[10] Bao J., Zheng Y., Mokbel M. F., Location-based and preference-aware recommendation using sparse geo-social networking data, Proceedings of the 20th International Conference on Advances in Geographic Information Systems, (2012), November 6-9; Redondo Beach, USA, pp.199-208, DOI: https://doi.org/10.1145/2424321.2424348

[11] Liu X., Liu Y., Aberer K., Miao C., Personalized point-of-interest recommendation by mining users' preference transition, Proceedings of the 22nd ACM international conference on Information \& Knowledge Management (CIKM '13), (2013), October 27-November 1; San Francisco, USA, pp.733-738, DOI: https://doi.org/10.1145/2505515.2505639

[12] Zhao Y. L., Nie L., Wang X., Chua T. S., Personalized recommendations of locally interesting venues to tourists via cross-region community matching, ACM Transactions on Intelligent Systems \& Technology, (2014), Vol.5, No.3, pp.1-26, DOI: https://doi.org/10.1145/2532439

[13] Hu L. K., Sun A. X., Liu Y., Your neighbors affect your ratings: on geographical neighborhood influence to rating prediction, Proceedings of the 37th international ACM SIGIR conference on Research \& development in information retrieval, (2014), July 25-30; Queensland, Australia, pp.345-354, DOI: https://doi.org/10.1145/2600428.2609593

[14] Ye M., Yin P. F., Lee W. C., Location recommendation for location-based social networks, Proceedings of the 18th SIGSPATIAL International Conference on Advances in Geographic Information Systems, (2010), November 2-5; San Jose, USA, pp.458-461, DOI: https://doi.org/10.1145/1869790.1869861

[15] Cao K., Guo J., Meng G., Liu H., Liu Y., Li G., Points-of-interest recommendation algorithm based on LBSN in Edge computing environment, IEEE Access, (2020), Vol.8, pp.47973-47983, DOI: 10.1109/ACCESS.2020.2979922 